\title{
Design and Implementation of an Image CoProcessor
}

\author{
R. Ebrahimi Atani ${ }^{1}$, S. Mirzakuchaki ${ }^{1}$, and S. Ebrahimi Atani ${ }^{2}$ \\ ${ }^{1}$ EE Department, IUST, Narmak 16846, Tehran, Iran \\ rebrahimi@iust.ac.ir, M_kuchaki@iust.ac.ir \\ http://webpages.iust.ac.ir/rebrahimi/ \\ 2 Department of Mathematics, Guilan University, \\ Faculty of Science, P.O. Box 1914, Rasht, Iran \\ ebrahimi@guilan.ac.ir
}

\begin{abstract}
This paper presents a novel DA based 2D DCT/DST coprocessor architecture for the synchronous design in a Xilinx FPGA device. A $1.2 \mathrm{~V}, 90 \mathrm{~nm}$ triple-oxide technology, Virtex-IV FPGA is used for final implementation and maximum operating frequency of $117 \mathrm{MHz}$ is achieved. Using XPower toolbox, the total dynamic power consumption of $393 \mathrm{~mW}$ is measured. The paper presents the trade-offs involved in designing the architecture, and the design for performance issues.
\end{abstract}

\section{Introduction}

Field programmable gate array (FPGA) digital signal processing (DSP) systems are increasingly employed by designers to address demanding digital communications, video, and imaging applications. Usually, FPGAs are used as VLSI replacement on low volume production or prototyping devices which are to be eventually implemented as application specific ICs (ASIC). The latest DSP applications demand system architecture solutions that address a range of requirements including performance, flexibility and scalability. FPGA coprocessors can offload a DSP processor and efficiently execute computationally intensive blocks of a DSP algorithm due to the inherent parallelism. This is especially attractive for emerging applications such as video and image processing and high speed digital communications where DSP performance requirements are growing at the fastest rates. The designer of DA based DSP algorithms in FPGAs is confronted with more design choices than the DSP programmer faces with the standard DSP microprocessor chips. Indeed, the number formats of the DSP microprocessor are pre-ordained either fixed point 16 or 24 bit, or 32 bit floating point word lengths. The FPGA design choice ranges from 2 bits to as many bits as are required; data word size and coefficient word size may be independently set, and may be modified along the data path all subject to the constraint of a fixed point number format. The choices available to the FPGA designer present both challenges and opportunities. Designs can be optimized in both performance and number of gate and memory resources.

A. Elmoataz et al. (Eds.): ICISP 2008, LNCS 5099, pp. 498 507, 2008.

(C) Springer-Verlag Berlin Heidelberg 2008 
Digital signal processing transforms (DXT) are required in almost all the phases of image and signal processing applications. DXTs are employed for various imaging techniques and spectral analysis of the signals. The discrete cosine transform (DCT) and discrete Sine transforms are two very well known DXTs that are widely used for VLSI digital signal and image processing systems. These two transforms have been used in image and speech processing and are the optimum and fast algorithms for image and data compression applications. DCT forms a key role in many image and video compression standards including JPEG2000 (image compression), ITU H.261 and ITU H.263 (Tele-conferencing), and ISO MPEG1 and MPEG2 (home video) [1]. DCT is also widely considered to be the most effective scheme for transform coding. DST is useful for spectrum analysis, data compression, speech processing, biomedical signal processing and in many other applications.

Several algorithms and implementations have been proposed for the DXTs, spreading from software implementations in DSPs to hardware implementations in $\operatorname{ASICs}($ 1], 2, [3, 7], and [10]). The DXTs are computationally intensive and as such, there is a great demand for high speed, high throughput and short latency computing architectures. Due to the high computation requirements, 2D DCT/DST processor design has been concentrated on small non overlapping blocks (typical $8 \times 8$ or $16 \times 16$ ). Many $2 \mathrm{D} \mathrm{DCT} / \mathrm{DST}$ algorithms have been proposed to achieve reduction of the computational complexity and thus increase the operational speed and throughput. The various algorithms and architectures for the 2D DCT can be divided into two categories:

1. Row/Column decomposition methods

2. Non row/column decomposition methods

There are some implementations for the DXT calculations, but two of the more important ones are systolic architecture (SA) and distributed arithmetic (DA). The main goal of the paper is the design of an $8 \times 8$ DA-based DCT/DST coprocessor. In this paper we will use DA due to their suitability for FPGA implementation and because they show dramatic improvements and better performance in comparison with the SA technique (in terms of speed and area consumption). We will also exploit row/column decomposition for parallelization and pipelining of the architecture. Paper presents the trade-offs involved in designing the architecture, and the design for performance issues.

This paper is structured as follows: a brief overview of DA is presented in Section 2. 1D DA based DCT/DST core architectures are described in section 3 . In section 4, 2D DCT/DST coprocessor design flow is presen ted. Implementation results are discussed in Section 5, and finally, conclusions are in Section 6.

\section{Distributed Arithmetic}

Distributed arithmetic is a bit level rearrangement of a multiply accumulate to hide the multiplications. DA plays a key role in embedding DSP functions in FPGA devices because the algorithm is based on Look up table (LUT). DA is 


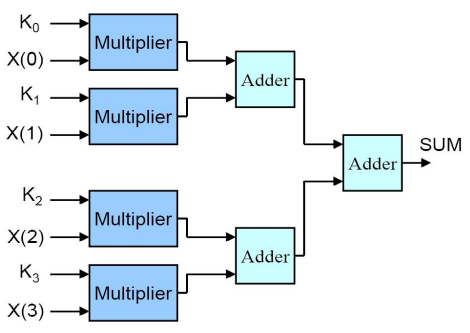

(i)

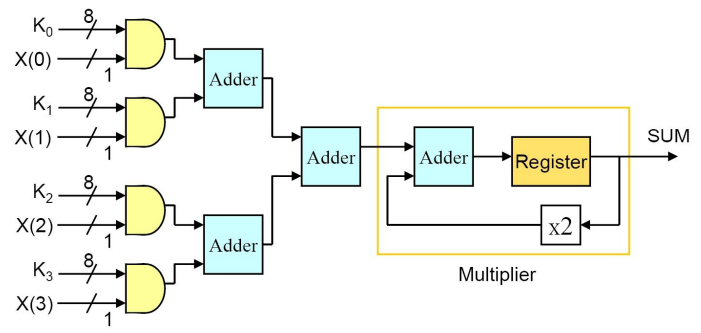

(ii)

Fig. 1. (i) 4-product MAC using Conventional Arithmetic (ii) 4-product MAC using Serial Distributed Arithmetic

one of the most common techniques where the multiply - accumulate (MAC) is of paramount importance. Its greatest advantage is in using additions instead of multiplications which is desirable since a multiplication consumes much more hardware than an addition. We can implement the 4-product MAC in two ways: one using conventional arithmetic and the other with serial DA as is shown in Fig. 1(i, ii). It is clearly obvious that great reductions on the hardware will be achieved when using DA. So In our implementation we have employed the 4 product MAC using Serial DA. The 4-product MACs, each consists of four multiplication units that are added together to produce the SUM output as illustrated in Fig. 1(i). In this design, the architecture in Fig. 1(ii) is used which produces the same result.

\section{1D DA Based DCT/DST Architecture}

Since the 1D DCT/DST are the basic elements for the 2D DCT/DST implementation, first the DCT and DST transforms of $1 \mathrm{D}$ will be presented. In case of $1 \mathrm{D}$, for any real sample sequence $x(n)$ where $n \in(0,1, \cdots, N-1)$, the DCT and DST can be defined as:

$$
\begin{aligned}
& D C T:\left\{\begin{array}{l}
C(k)=\sqrt{\frac{2}{N}} \cdot \alpha_{k} \sum_{n=0}^{N-1} x(n) \cos \left[(2 n+1) \cdot \frac{k \cdot \pi}{2 N}\right] \\
k=0,1, \cdots, N-1 \text { where } \alpha_{k}= \begin{cases}\frac{1}{\sqrt{2}} & k=0 \\
1 & \text { otherwise }\end{cases}
\end{array}\right. \\
& D S T:\left\{\begin{array}{l}
S(k)=\sqrt{\frac{2}{N}} \cdot \beta_{k} \sum_{n=0}^{N-1} x(n) \sin \left[(2 n+1) \cdot \frac{k \cdot \pi}{2 N}\right] \\
k=1,2, \cdots, N \text { where } \beta_{k}= \begin{cases}\frac{1}{\sqrt{2}} & k=N \\
1 & \text { otherwise }\end{cases}
\end{array}\right.
\end{aligned}
$$

The direct implementation of these equations need $N^{2}$ multiplications. Because cyclic properties and symmetry of sinusoidal functions, the number of 
multiplications can be reduced. According to the definition of DCT in (1), a different representation for the DCT data sequence is shown in (3).

$$
C(k)=\sum_{n=0}^{N-1} A_{n, k} \cdot x(n)
$$

Where, $A$ is the transform matrix. The real-time computation of DCT requires a lot of calculations because of the large number of multiplications involved, therefore much effort has gone into reducing the total number of multiplications. Various authors have proposed diverse algorithms for this reduction, like matrix decomposition, polynomial transformation or combinations of both. By using matrix decomposition and taking advantage of the coefficient symmetry from Chen et al [6, it can be shown that we can divide the transform matrix $A$ into two smaller matrices, significantly reducing the total number of multiplications to $\frac{N^{2}}{2}$. Then DCT is obtained easily from (4) and (5):

$$
\begin{aligned}
C(k) & =\sum_{n=0}^{\frac{N}{2}-1} A_{n, k} \cdot[x(n)+x(N-1-n)] \quad \text { for even } k \\
C(k) & =\sum_{n=0}^{\frac{N}{2}-1} A_{n, k} \cdot[x(n)-x(N-1-n)] \quad \text { for odd } k
\end{aligned}
$$

In case of an 8-point transform, due to the symmetry, $A$ can be replaced by two matrices which can be computed in parallel, according to (6) and (7):

$$
\left[\begin{array}{l}
Y(0) \\
Y(2) \\
Y(4) \\
Y(6)
\end{array}\right]=\left[\begin{array}{cccc}
0.5 \cos \left(\frac{\pi}{4}\right) & 0.5 \cos \left(\frac{\pi}{4}\right) & 0.5 \cos \left(\frac{\pi}{4}\right) & 0.5 \cos \left(\frac{\pi}{4}\right) \\
0.5 \cos \left(\frac{\pi}{8}\right) & 0.5 \cos \left(\frac{3 \pi}{8}\right) & -0.5 \cos \left(\frac{3 \pi}{8}\right) & -0.5 \cos \left(\frac{\pi}{8}\right) \\
0.5 \cos \left(\frac{\pi}{4}\right) & -0.5 \cos \left(\frac{\pi}{4}\right) & -0.5 \cos \left(\frac{\pi}{4}\right) & 0.5 \cos \left(\frac{\pi}{4}\right) \\
0.5 \cos \left(\frac{3 \pi}{8}\right) & -0.5 \cos \left(\frac{\pi}{8}\right) & 0.5 \cos \left(\frac{\pi}{8}\right) & -0.5 \cos \left(\frac{3 \pi}{8}\right)
\end{array}\right]\left[\begin{array}{l}
x(0)+x(7) \\
x(1)+x(6) \\
x(2)+x(5) \\
x(3)+x(4)
\end{array}\right]
$$

$$
\left[\begin{array}{l}
Y(1) \\
Y(3) \\
Y(5) \\
Y(7)
\end{array}\right]=\left[\begin{array}{cccc}
0.5 \cos \left(\frac{\pi}{16}\right) & 0.5 \cos \left(\frac{3 \pi}{16}\right) & 0.5 \cos \left(\frac{5 \pi}{16}\right) & 0.5 \cos \left(\frac{7 \pi}{16}\right) \\
0.5 \cos \left(\frac{3 \pi}{16}\right) & -0.5 \cos \left(\frac{7 \pi}{16}\right) & -0.5 \cos \left(\frac{\pi}{16}\right) & -0.5 \cos \left(\frac{5 \pi}{16}\right) \\
0.5 \cos \left(\frac{5 \pi}{16}\right) & -0.5 \cos \left(\frac{\pi}{16}\right) & -0.5 \cos \left(\frac{7 \pi}{16}\right) & 0.5 \cos \left(\frac{3 \pi}{16}\right) \\
0.5 \cos \left(\frac{7 \pi}{16}\right) & -0.5 \cos \left(\frac{5 \pi}{16}\right) & 0.5 \cos \left(\frac{3 \pi}{16}\right) & -0.5 \cos \left(\frac{\pi}{16}\right)
\end{array}\right]\left[\begin{array}{l}
x(0)-x(7) \\
x(1)-x(6) \\
x(2)-x(5) \\
x(3)-x(4)
\end{array}\right]
$$

It should be noted that the calculation of DST is similar to DCT with the only distinction being the different coefficient matrices and we are not going into details about it any further. (8) and (9) show the DST coefficients. Fig. 2 illustrates the overall architectures for 8-point 1D DCT and 1D DST. The four sum boxes are the equivalents of the corresponding sums in parenthesis in (6) and (9) and also the four difference boxes are coming from differences in (7) and (8). In the next stage we have 4-product MACs which each one consists of four multiplication units that are added together to produce the SUM output. We can implement the 4-product MAC using serial distributed arithmetic as illustrated 


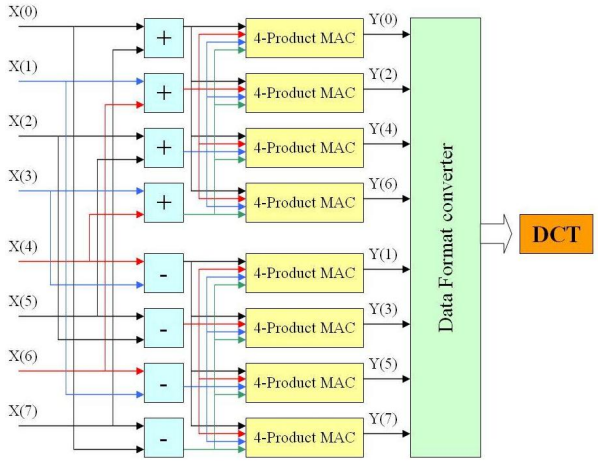

(i)

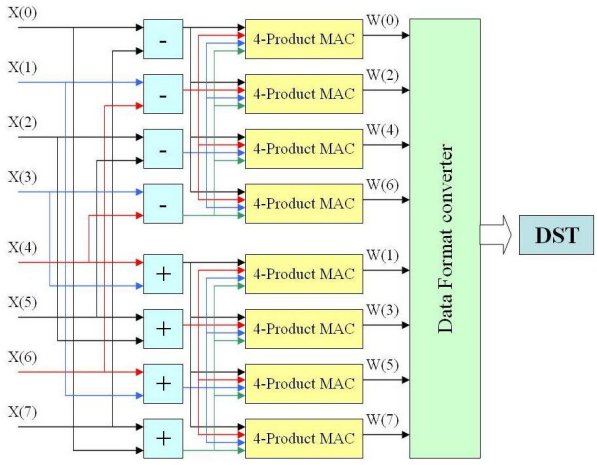

(ii)

Fig. 2. (i) 8-point 1-D DCT (ii) 8-point 1-D DST

in Fig. 1 (ii). If we examine (6) and (7) again, we can observe that the coefficient matrices are $4 \times 4$ and that is why we have used 4-product MACs. Each 4 -product MAC calculates the multiplication of two vectors: one row of the coefficient matrix and the other vector consisted of four sum boxes (or difference boxes). The outputs of the MACs are not in the correct order and need reordering. In the final stage there is a Data Format Converter that has the task of re-arranging the outputs of the MACs and delivers the final DCT or DST result.

$$
\left[\begin{array}{l}
W(8) \\
W(2) \\
W(4) \\
W(6)
\end{array}\right]=\left[\begin{array}{cccc}
0.5 \sin \left(\frac{\pi}{4}\right) & 0.5 \sin \left(\frac{\pi}{4}\right) & 0.5 \sin \left(\frac{\pi}{4}\right) & 0.5 \sin \left(\frac{\pi}{4}\right) \\
0.5 \sin \left(\frac{\pi}{8}\right) & 0.5 \sin \left(\frac{3 \pi}{8}\right) & -0.5 \sin \left(\frac{3 \pi}{8}\right) & -0.5 \sin \left(\frac{\pi}{8}\right) \\
0.5 \sin \left(\frac{\pi}{4}\right) & -0.5 \sin \left(\frac{\pi}{4}\right) & -0.5 \sin \left(\frac{\pi}{4}\right) & 0.5 \sin \left(\frac{\pi}{4}\right) \\
0.5 \sin \left(\frac{3 \pi}{8}\right) & -0.5 \sin \left(\frac{\pi}{8}\right) & 0.5 \sin \left(\frac{\pi}{8}\right) & -0.5 \sin \left(\frac{3 \pi}{8}\right)
\end{array}\right]\left[\begin{array}{l}
x(0)-x(7) \\
x(1)-x(6) \\
x(2)-x(5) \\
x(3)-x(4)
\end{array}\right]
$$

$$
\left[\begin{array}{l}
W(1) \\
W(3) \\
W(5) \\
W(7)
\end{array}\right]=\left[\begin{array}{cccc}
0.5 \sin \left(\frac{\pi}{16}\right) & 0.5 \sin \left(\frac{3 \pi}{16}\right) & 0.5 \sin \left(\frac{5 \pi}{16}\right) & 0.5 \sin \left(\frac{7 \pi}{16}\right) \\
0.5 \sin \left(\frac{3 \pi}{16}\right) & -0.5 \sin \left(\frac{7 \pi}{16}\right) & -0.5 \sin \left(\frac{\pi}{16}\right) & -0.5 \sin \left(\frac{5 \pi}{16}\right) \\
0.5 \sin \left(\frac{5 \pi}{16}\right) & -0.5 \sin \left(\frac{\pi}{16}\right) & -0.5 \sin \left(\frac{7 \pi}{16}\right) & 0.5 \sin \left(\frac{3 \pi}{16}\right) \\
0.5 \sin \left(\frac{7 \pi}{16}\right) & -0.5 \sin \left(\frac{5 \pi}{16}\right) & 0.5 \sin \left(\frac{3 \pi}{16}\right) & -0.5 \sin \left(\frac{\pi}{16}\right)
\end{array}\right]\left[\begin{array}{l}
x(0)+x(7) \\
x(1)+x(6) \\
x(2)+x(5) \\
x(3)+x(4)
\end{array}\right]
$$

The reduction in hardware in distributed arithmetic is evident from the previous figures. We have used ROMs to store the coefficients needed, speeding up the transform calculation by a large degree. But using ROMs has the disadvantage of adding to the hardware cost, which is undesirable. It can be ameliorated by using the CORDIC algorithm to calculate the coefficients but in real-time applications such as video and image processing it is not feasible and the use of the CORDIC algorithm is left for special CORDIC processors where speed is not vital. 


\section{2D DCT/DST Architecture and Design Flow}

In this section the design flow of the image coprocessor is discussed. The equations for the $N \times N$ point 2D DXT of an input sequence $\{x(m, n): m=n=$ $0,1, \cdots, N-1\}$ can be shown as:

$$
\begin{aligned}
& 2 D D C T:\left\{\begin{array}{l}
C(k, l)=\sqrt{\frac{2}{N}} \cdot \alpha_{k} \alpha_{l} \sum_{n=0}^{N-1} \sum_{m=0}^{N-1} x(m, n) \cos \left[(2 m+1) \cdot \frac{k \cdot \pi}{2 N}\right] \cdot \cos \left[(2 n+1) \cdot \frac{l \cdot \pi}{2 N}\right] \\
k, l=0,1, \cdots, N-1 \text { where } \alpha_{k}=\alpha_{l}= \begin{cases}\frac{1}{\sqrt{2}} & k=l=0 \\
1 & \text { otherwise }\end{cases}
\end{array}\right. \\
& 2 D D S T:\left\{\begin{array}{l}
S(k, l)=\sqrt{\frac{2}{N}} \cdot \beta_{k} \beta_{l} \sum_{n=0}^{N-1} \sum_{m=0}^{N-1} x(m, n) \sin \left[(2 m+1) \cdot \frac{k \cdot \pi}{2 N}\right] \cdot \sin \left[(2 n+1) \cdot \frac{l \cdot \pi}{2 N}\right] \\
k, l=0,1, \cdots, N-1 \text { where } \beta_{k}=\beta_{l}= \begin{cases}\frac{1}{\sqrt{2}} & k=l=0 \\
1 & \text { otherwise }\end{cases}
\end{array}\right.
\end{aligned}
$$

It is easy to see that, direct implementation of (10) or (11) needs $N^{4}$ multiplications. But in real time processing with hardware requirements and energy consumption considerations, this is infeasible. Reordering (10) and (11), it can be broken down in two groups of $N$ 1D-DXT, which is equivalent to processing a data block by rows, followed by a column processing or vice versa. This is the most widely utilized method due to its simplicity, regular form and easy implementation. By using matrix notation, the $N \times N$ point 2D DXT of (10) or (11) can be expressed as a matrix vector computation:

$$
[T]=\left[M_{N \times N}\right] \cdot[x]
$$

Where $M$ represents the DXT coefficient matrix. By applying row column decomposition, the algorithm can be rewritten using two 1D DXTs and a matrix transpose, as shown in (13):

$$
[T]=\left[Q_{1 \times N}\right] \cdot[x] \cdot\left[Q_{N \times 1}\right]^{t}
$$

This method needs a transposition memory between the two 1D DXT blocks. If the timing specifications of the application permit it, only one $N$ 1D DXT block can be used for both row and column processing. It is necessary to add a multiplexer for the selection of the input to the DXTs, but the $N$ 1D DXT area is saved. The drawback of this method is the limited bandwidth of transposition memory. In the design of image coprocessor two 8 point $1 \mathrm{D}$ DXT is used and $8 \times 82 \mathrm{D}$ DXT is applied to the input image.

\subsection{Design Strategy}

In this section we will describe the design strategy of the 2D image coprocessor. The transformation core has simple input interface. Design is synchronous and whole architecture use two 1D DCT and two 1D DST blocks to produce 2D 


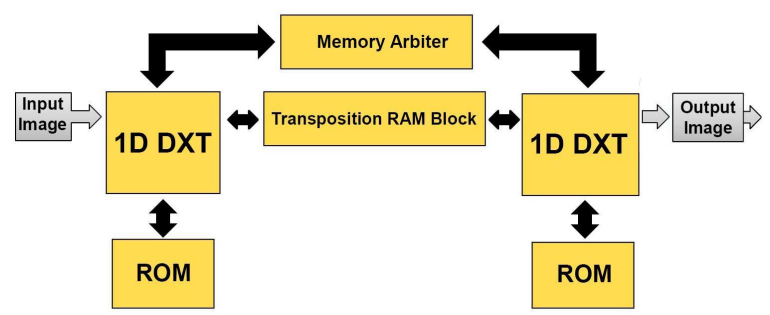

Fig. 3. Architecture for 2D DXT coprocessor

trasforms. A multiplexer is used to distingush between DST and DCT transforms. The block diagram of the whole architecture is shown in Fig. 3. Two 1D DXT units are connected through transposition matrix memory. Latency between first latched input data and first DXT transformed output is 85 clock cycles. Design is internally pipelined, when the pipeline is full 64 point input data is transformed in 64 clock cycles to $2 \mathrm{D}$ DXT values.

Core uses double buffered RAM for storing intermediate product results after first DXT stage for maximized performance. This way both 1D DXT units can work in parallel effectively creating dual stage global pipeline. 2D DXT core takes 8 bit input data and produces 12 bit output using 12 bit DXT matrix coefficients. This may be enhanced to be configurable in the future. Transposition RAM is double buffered, that is when second stage of DXT reads out data from transposition memory 1, first DXT stage can populate second transposition memory with new data. This enables creation of dual stage global pipeline where every stage consists of 1D DXT and transposition memory. 1D DXT units are not internally pipelined; they use parallel DA to compute DXT values. Because of parallel DA they need considerable amount of ROM memories to compute one DXT values in single clock cycle. Design based on DA does not use any multipliers for computing MAC, instead it stores precomputed MAC results in ROM memory and grab them as needed. ROMs can be either asynchronous or synchronous. A memory arbiter between DXT stages is essentially needed. Each stage can request memory buffer for read/write and basing on availability one of two RAM buffers is granted. Every RAM has 10 bit width data and 64 memory cells. On chip RAMs used are dual port synchronous memory with one clock cycle delay. These RAMs are currently 64 words of 14 bits size and there are two of them to create ping pong buffers for improved throughput.

\subsection{Testbench}

In order to test the applicability of the architecture, a ModelSim based testbench is created. The testbench takes Lena image as input (using Matlab image to text function). Image coprocessor transforms the Lena image to 2D DCT or 2D DST matrices and then by ModelSIM and a behavioral inverse transform reconstructed image is obtained. To compare the quality of reconstruction, 


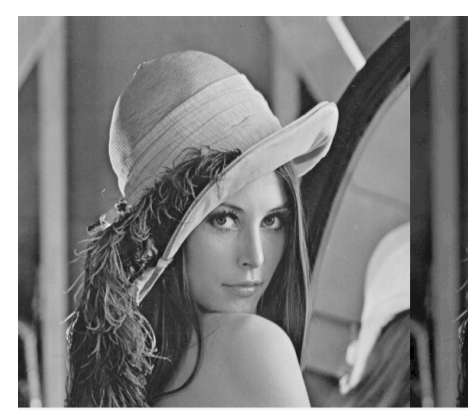

(i)

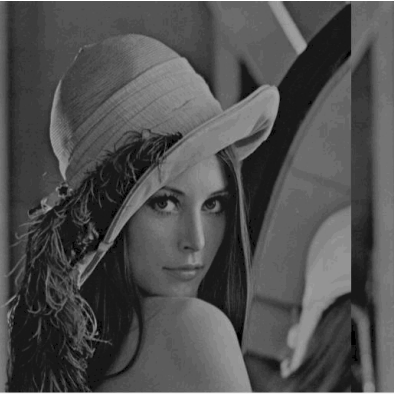

(ii)

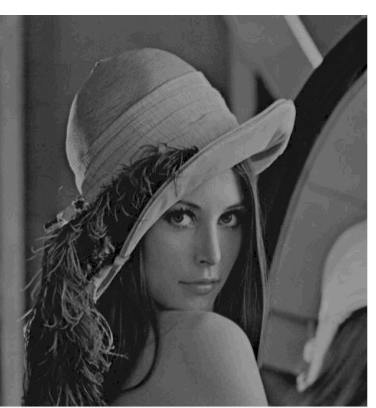

(iii)

Fig. 4. (i) Original image (ii) 2D-DCT reconstructed image (iii) 2D-DST reconstructed image

the peak signal to noise ratio (PSNR) is computed between original and reconstructed images. In case of $(512 \times 512)$ pixel size Lena image, PSNR of 2D-DCT and 2D-DST reconstructed images was $45.5 \mathrm{~dB}$ and $45 \mathrm{~dB}$ respectively. The obtained PSNRs are above the required margin of $40 \mathrm{~dB}$. The introduced errors are because of fixed point arithmetic operations. The original Lena image and reconstructed 2D-DCT/DST images are shown in Fig. 4.

\section{Implementation Results}

The whole architecture including the computation block, data path, and control unit is modeled at register transfer level in VHDL, simulated and tested by a testbench using Modelsim and Matlab. The target device for implementation is a $90 \mathrm{~nm}$ VertexIV Xilinx FPGA. The hardware description of this architecture was synthesized using ISE Xilinx 8.1 and mapped on the VertexIV (4VSX35FF66812). The timing analysis showed that, the worst delay time is less than $8.5 \mathrm{~ns}$ which set up the maximum clock of the system to $117 \mathrm{MHz}$. The architecture's set up slack is 1.546 nano seconds. $56 \%$ of data arrival time is because of cell

Table 1. Device Utilization for Vertex-IV (4VSX35FF668-12)

\begin{tabular}{|c||c|c|c|}
\hline Resource & Used & Available & Utilization \\
\hline \hline IOs & 33 & 448 & $7.37 \%$ \\
\hline Global Buffers & 1 & 32 & $3.13 \%$ \\
\hline Function Generators & 1012 & 30720 & $3.3 \%$ \\
\hline CLB Slices & 563 & 15360 & $3.7 \%$ \\
\hline Dffs or Latches & 579 & 31616 & $1.83 \%$ \\
\hline Block RAMs & 42 & 192 & $21.88 \%$ \\
\hline DSP48s & 0 & 192 & $0.00 \%$ \\
\hline
\end{tabular}


delay and the remaining $44 \%$ is due to net delay. Power consumption of the architecture has been estimated using XPower toolbox of ISE with an input stimuli showing the clocking and toggling rates of the architecture's components. The power summary showed that the total dynamic power dissipation of this implementation is $393 \mathrm{~mW}$. The resource utilization has been demonstrated in table 1. Less than $7 \%$ of the LUTs of the FPGA chip has been used.

\section{Summary and Conclusions}

This paper has proposed an efficient mapping on FPGA of an image coprocessor. A fully parallel architecture based on row/column decomposition is proposed for the computation of the $2 \mathrm{D}$ DA based $8 \times 8$ DCT/DST on a Vertex IV Xilinx FPGA. The system is highly modular and utilizes a highly parallel structure to achieve high speed performance. It uses two identical units for the computation of the row and column transforms. We have exploited DA in order to achieve surface reduction and precision amelioration compared to conventional algorithm.

\section{Acknowledgment}

Reza Ebrahimi Atani receives partial funding through Iran Telecommunication Research Center (http://www.itrc.ac.ir).

\section{References}

1. Amiri, M., Ebrahimi Atani, R., Mirzakuchaki, S., Mahdavi, M.: Design and Implementation of $50 \mathrm{MHz}$ DXT Coprocessor. In: 10th Euromicro Conference on Digital System Design, Architectures, Methods and Tools, August 29-31, 2007, pp. 43-50. IEEE press, Lubeck (2007)

2. EL-Bannai, A., EL-Fattah, A., Fakhr, W.: An efficient implementation of the 1D DCT using FPGA technology. In: 11th IEEE International Conference and Workshop on the Engineering of Computer-Based Systems, May 24-27, 2004, vol. 27, pp. 356-360 (2004)

3. Ebrahimi Atani, R., Mirzakuchaki, S., Samii, F., Nasrollahzadeh, M.: Design and Implementation of a $157 \mathrm{MHz}$ DA-Based DXT Coprocessor. In: 4th IEEGCC conference, Manama, Kingdom of Bahrain, November 11-14 (2007)

4. Ahmed, N., Natarajan, T., Rao, K.R.: Discrete cosine transform. IEEE Transactions on Computers C-23(1), 90-93 (1974)

5. Wu, H.R., Paoloni, F.J.: A two-dimensional fast cosine transform algorithm based on Hou's approach. IEEE Transactions on Signal Processing 39(2), 544-546 (1991)

6. Chen, W.H., Smith, C.H., Fralick, S.C.: A fast computational algorithm for the discrete Cosine transform. IEEE Transactions on Communications 25(9), 10041009 (1977)

7. Ghosh, S., Venigalla, S., Bayoumi, M.: Design and Implementation of a 2D-DCT Architecture using Coefficient Distributed Arithmetic. In: Proceedings of the IEEE Computer Society Annual Symposium on VLSI, May 11-12, 2005, pp. 162-166 (2005) 
8. Aggoun, A., Jalloh, I.: Two-dimensional DCT/IDCT architecture. In: IEE Proceedings Computers and Digital Techniques, January 2-10, 2003, vol. 150(1), pp. 2-10. IEEE Press, Los Alamitos (2003)

9. Baoyu, Z.: A New Algorithm for the 2D Discrete Cosine Transform. In: Fourth International Conference on Signal Processing Proceedings, October 12-16, 1998, pp. 85-88. IEEE Press, Los Alamitos (1998)

10. Samadi, P., Wu, H., Ahmadi, M.: The 2-D Quantized DCT With Distributed Arithmetic. In: Canadian Conference on Electrical and Computer Engineering, May 2006, pp. 2049-2052. IEEE Press, Ottawa (2006)

11. Gonzalez, R., Woods, C.: Digital Image Processing, 3rd edn. Pearson, Prentice Hall (2007) 